\title{
Calcification of Human Saphenous Vein Associated with Endothelial Dysfunction: A Pilot Histopathophysiological and Demographical Study
}

\author{
Sydney L. Pedigo ${ }^{1}$, Christy M. Guth ${ }^{1}$, Kyle M. Hocking ${ }^{1}$, Alex Banathy', Fan Dong Li', \\ Joyce Cheung-Flynn', Colleen M. Brophy ${ }^{1,2}$, Raul J. Guzman ${ }^{3}$ and Padmini Komalavilas,2* \\ ${ }^{1}$ Department of Surgery, Vanderbilt University Medical Center, Nashville, TN, USA, ${ }^{2}$ Veterans Affairs Tennessee Valley \\ Healthcare System, Nashville, TN, USA, ${ }^{3}$ Beth Israel Deaconess Medical Center, Boston, MA, USA
}

OPEN ACCESS

Edited by:

Faisal Aziz,

Penn State Milton S. Hershey

Medical Center, USA

Reviewed by:

Andreas M. Lazaris,

National and Kapodistrian University of Athens, Greece

Thodur Madabushi Vasudevan,

Waikato Hospital, New Zealand

*Correspondence:

Padmini Komalavilas padmini.komalavilas@vanderbilt.edu

Specialty section: This article was submitted to Vascular Surgery,

a section of the journal

Frontiers in Surgery

Received: 16 August 2016 Accepted: 23 January 2017 Published: 09 February 2017

Citation: Pedigo SL, Guth CM, Hocking KM, Banathy A, Li FD, Cheung-Flynn J,

Brophy CM, Guzman RJ and Komalavilas P (2017) Calcification of Human Saphenous Vein Associated with Endothelial Dysfunction: A Pilot Histopathophysiological and Demographical Study. Front. Surg. 4:6.

doi: 10.3389/fsurg.2017.00006
While the pathophysiology and clinical significance of arterial calcifications have been studied extensively, minimal focus has been placed on venous calcification deposition. In this study, we evaluated the association between calcium deposition in human saphenous vein (HSV), endothelial function, and patient demographic risk factors. Fifty-four HSV segments were collected at the time of coronary artery bypass graft (CABG) surgery. The presence or absence of calcium deposits was visualized using the Von Kossa staining method. Endothelial function was determined by measuring muscle tissue contraction with phenylephrine and relaxation with carbachol in a muscle bath. Additional segments of vein underwent histologic evaluation for preexisting intimal thickness and extracellular matrix (ECM) deposition. Patient demographics data were obtained through our institution's electronic medical record, with patient consent. Calcium was present in 16 of 54 samples (29.6\%). Veins with calcium deposits had significantly greater intimal-to-medial thickness ratios $(p=0.0058)$ and increased extracellular collagen deposition ( $p=0.0077$ ). Endothelial relaxation was significantly compromised in calcified veins vs. those without calcium $(p=0.0011)$. Significant patient risk factors included age $(p=0.001)$ and preoperative serum creatinine $(p=0.017)$. Calcified veins can be characterized as having endothelial dysfunction with increased basal intimal thickness and increased ECM deposition. Patient risk factors for calcium deposits in veins were similar to those for arteries, namely, advanced age and kidney disease. Further studies are needed to determine the effect of preexisting vein calcification on short- and longterm graft patency.

Keywords: calcification, demographics, intimal thickness, endothelial dysfunction, endothelial-dependent relaxation, vein graft failure, human saphenous vein

\section{INTRODUCTION}

Saphenous vein is the most commonly used bypass conduit for coronary artery and peripheral arterial bypass surgery. However, recent large prospective studies have indicated that graft failure rates remain high [39\% of lower extremity bypass grafts and $45 \%$ of coronary artery bypass grafts (CABGs) 
will fail within 12-18 months, Project of Ex vivo Vein Graft Engineering via Transfection (PREVENT) III and PREVENT IV trials, respectively] $(1,2)$. The leading cause of graft failure is intimal hyperplasia ( $\mathrm{IH}$ ), a complex process involving the migration, proliferation, and phenotypic modulation of vascular smooth muscle cells (VSMCs) (3).

Preexisting disease in the saphenous vein has been implicated as a contributing factor to the development of IH and graft failure. The most frequently encountered lesion found on pre-bypass vein histology is intimal thickening (4). Our lab has previously published an association between preexisting intimal thickness and endothelial dysfunction (5). Marin et al. reported more frequent peripheral bypass graft failure with use of thicker-walled saphenous vein (6). Additional characteristics in morphology may contribute to the baseline quality of a saphenous vein graft.

Studies conducted to determine the quality of arterial conduits for CABG (most commonly the internal mammary and radial arteries) have evaluated vessel wall thickness and vessel wall calcification (7-9). In general, the pathophysiology and clinical relevance of arterial calcification is much better established than for venous calcification. Arterial calcification is broadly categorized by its vascular layer of involvement. Medial calcification, or Monckeberg sclerosis, is generally associated with metabolic derangement, such as in type 2 diabetes mellitus, end-stage renal disease, and hyperphosphatemia, and results in reduced arterial compliance and elasticity (10-12). The subsequent widening of pulse pressure is an independent predictor of hypertension, stroke, and all-cause mortality. In contrast, intimal calcification, a component of atherosclerosis, is more often associated with inflammatory states, such as in hyperlipidemia, hypertension, and smoking. These calcified plaques contribute to the narrowing of the arterial lumen and increase the risk of ischemic events. Coronary artery calcification of either type is an independent predictor of cardiovascular mortality and is used as a surrogate end point for clinical trials to determine the efficacy of medications and lifestyle modification (13). Since saphenous vein is the most widely used conduit for CABG, a study of vein calcification will help to understand how it affects the quality of the vein as a conduit and whether it contributes to the development of IH and subsequent graft failure.

In this study, we evaluated saphenous vein segments, obtained at the time of CABG surgery, for preexisting calcification. The presence or absence of calcium deposits was then correlated with other histologic markers of venous pathology (intimal thickness and ECM deposition), physiologic function, and patient demographics. We hypothesized that the presence of calcium deposits would be associated with concurrent venous pathology and physiologic dysfunction. In addition, we anticipated that known patient risk factors for arterial calcification would also predict the presence of venous calcification.

\section{MATERIALS AND METHODS}

\section{Procurement of Human Saphenous Veins (HSVs)}

Human saphenous vein samples were collected at the time of harvest for CABG surgery. This study was carried out in accordance with the recommendations of the Institutional Review Boards of Vanderbilt University Medical Center and the VA Tennessee Valley Healthcare System in Nashville, TN, USA with written informed consent from all subjects. All subjects gave written informed consent in accordance with the Declaration of Helsinki. The protocol was approved by the Institutional Review Boards of Vanderbilt University Medical Center and the VA Tennessee Valley Healthcare System in Nashville, TN, USA. Segments of vein were harvested from 54 patients by either open or endoscopic technique, according to surgeon preference. Samples were immediately stored in sterile, heparinized $(10 \mathrm{U} / \mathrm{mL})$ PlasmaLyte (Baxter Healthcare Corporation, Deerfield, IL, USA) solution (140 mEq sodium, $5 \mathrm{mEq}$ potassium, $3 \mathrm{mEq}$ magnesium, $98 \mathrm{mEq}$ chloride, $27 \mathrm{mEq}$ acetate, and $23 \mathrm{mEq}$ gluconate) without further manipulation. Tissue with obvious forceps crush injury and areas containing a tributary orifice were discarded. The remaining tissue was then cut into sequential, $2 \mathrm{~mm}$ rings, fixed with $10 \%$ buffered formalin, and sent to the Translational Pathology Shared Resource at Vanderbilt University Medical Center for histologic preparation of $5 \mu \mathrm{m}$ sequential sections embedded in paraffin. Additional fresh tissue rings were cut and suspended in a muscle bath to evaluate physiologic properties.

\section{Evaluation of Calcium Deposits}

Vein ring sections were stained using the Von Kossa method to observe calcification. Paraffin was melted and removed from each slide. Slides were then incubated in 5\% silver nitrate solution for $2 \mathrm{~h}$ under a $50 \mathrm{~W}$ light (control slide was incubated in water). Slides were then washed with $5 \%$ sodium thiosulfate, counterstained with hematoxylin, dehydrated, and mounted. Tissue images were obtained and digitalized using the Zeiss Axiovert $200 \mathrm{M}$ microscope at $20 \times$ magnification. Veins were then grouped as having no calcium deposits (non-calcified) or having some amount of calcium deposits present (calcified).

\section{Intimal Thickness Measurement}

Verhoeff-Van Gieson (VVG) stain was used to visualize the vein intima and media. A total of eight intimal width measurements were made from each image, two from each quadrant at $20 \times$. Vein intimal thickness for each sample was defined as the mean of these measurements. The medial thickness was obtained in a similar fashion at $5 \times$ magnification, and the intimal/medial ratio (IMR) was calculated.

\section{Extracellular Matrix (ECM) Deposition Analysis}

Extracellular matrix deposition was examined using a Movat pentachrome stain, which gives collagen a yellow-brown hue. The collagen amount was quantified using Photoshop C5's pixel count feature at a fuzziness level of $50 \%$. The pixel count was standardized as a ratio to the overall image pixel number. At least four pictures from each vein sample were analyzed and the ratios averaged.

\section{Physiologic Measurements of HSVs}

Details regarding the use of muscle baths to determine vascular physiologic function have been previously described (5). 
Briefly, $1 \mathrm{~mm}$ rings from the tissue were cut, weighed, and measured lengthwise using calipers. Rings were suspended in an organ bath containing a bicarbonate buffer $(120 \mathrm{mM}$ $\mathrm{NaCl}, 4.7 \mathrm{mM} \mathrm{KCl}, 1.0 \mathrm{mM} \mathrm{MgSO}_{4}, 1.0 \mathrm{mM} \mathrm{NaH}_{2} \mathrm{PO}_{4}, 10 \mathrm{mM}$ glucose, $1.5 \mathrm{mM} \mathrm{CaCl}_{2}$, and $25 \mathrm{mM} \mathrm{Na}_{2} \mathrm{HCO}_{3}$, pH 7.4), equilibrated with $95 \%$ oxygen and $5 \%$ carbon dioxide at $37^{\circ} \mathrm{C}$. Each ring was progressively stretched to its optimal resting tension (approximately $1 \mathrm{~g}$ ) that would produce a maximal response to contractile agonists, then maintained at the resting tension and equilibrated for a minimum of $2 \mathrm{~h}$. Force measurements were obtained using a Radnoti Glass Technology (Monrovia, CA, USA) force transducer (159901A) interfaced with a Powerlab data acquisition system and Chart software (ADInstruments, Colorado Springs, CO, USA). The rings were contracted with $110 \mathrm{mM}$ potassium chloride $(\mathrm{KCl})$ to determine functional viability. Samples that failed to contract with $\mathrm{KCl}$ were considered non-functional and removed from further testing. Functional rings were equilibrated in the bicarbonate buffer for $30 \mathrm{~min}$ and were then contracted with phenylephrine $\left(10^{-6} \mathrm{M}\right)$, and the endothelium-dependent relaxation was measured with carbachol $\left(5 \times 10^{-7} \mathrm{M}\right)$, a cholinergic agonist that causes tissue relaxation in the presence of intact endothelium. The force generated was measured and converted to stress $\left\{\left(10^{5} \mathrm{~N} /\right.\right.$ $\left.\mathrm{m}^{2}\right)=$ force $(\mathrm{g}) \times 0.0987 /$ area, where area is equal to the wet weight $[(\mathrm{mg}) /$ length $(\mathrm{mm}$ at maximal length) $]$ divided by $1.055\}$ using each specimen's weight and length. The percentage of endothelial-dependent relaxation was calculated as the change in stress compared to the maximal tension induced by phenylephrine as previously described (14).

\section{Patient Demographic Collection}

Patient demographics and lab values were obtained using institutional electronic medical records with patient consent. The data collected included demographic characteristics, laboratory results, comorbidities, and medications listed at the time of surgery. Patients were grouped by the presence or absence of preexisting saphenous vein calcification.

\section{Statistical Analysis}

Bivariate analysis of all variables was conducted to screen for those factors associated with calcification (via Pearson chi-squared test). Factors that trended with calcification were included in logistic multivariate stepwise regression models. Variables significant upon multivariate analysis were deemed independent predictors of the end points, at a level of statistical significance of $p<0.05$. As all variables included in multivariate analyses had a variance inflation factor of $<1.8$, collinearity was not statistically considered. Measures of central tendency were reported as mean $\pm \mathrm{SD}$. Statistical analysis was performed with JMP Pro 11 (Cary, NC, USA) and GraphPad Prism (La Jolla, CA, USA).

\section{RESULTS}

A total of 54 vein segments were collected and analyzed for the presence of calcification. Forty of these samples underwent additional histologic analysis to evaluate preexisting intimal thickness and ECM deposition. The remaining 14 segments were excluded due to improper histologic processing. All 54 vein segments underwent physiologic testing on muscle baths. Of these, 39 were viable based on the $\mathrm{KCl}$ contraction challenge. Finally, we obtained baseline demographic data on 53 patients.

\section{Presence of Calcium Associated with Preexisting Intimal Thickness}

Calcium deposits were present in 16 of 54 vein samples (29.6\%) (Figure 1). Calcified veins had greater preexisting intimal thickness, expressed IMR, compared to non-calcified veins $(0.214 \pm 0.028$ vs. $0.134 \pm 0.013 ; p=0.006)$ (Figure 2$)$

\section{Calcification Associated with Additional Markers of Vascular Pathology}

We examined whether calcified veins would exhibit other histologic markers of pathology. ECM collagen deposition is a component of the IH response (15). Calcified veins revealed more ECM collagen deposition, expressed as a pixel ratio, overall $(0.210 \pm 0.029$ vs. $0.111 \pm 0.020 ; p=0.0077)$. It was notable that collagen deposition appeared to localize with calcium with areas of increased calcium and collagen density within the tissue ring (Figure 3).

\section{Venous Calcification Inversely Correlated with Endothelial-Dependent Relaxation}

We then examined whether calcification affected the endothelial function of a vein. One reliable method of assessing vessel function is endothelial relaxation. Vein rings were contracted with phenylephrine $\left(10^{-6} \mathrm{M}\right)$ and treated with carbachol $\left(5 \times 10^{-7} \mathrm{M}\right)$. In the presence of an intact, functioning endothelium, carbachol induces relaxation of the vessel. Thirty-nine vein samples were determined to be viable, and the percentage of carbachol-induced relaxation was calculated. The endothelial relaxation of calcified veins was lower in comparison to non-calcified veins $(3.3 \pm 0.9$ vs. $18.4 \pm 3.0 \%, p=0.0011$, Figure 4 ).

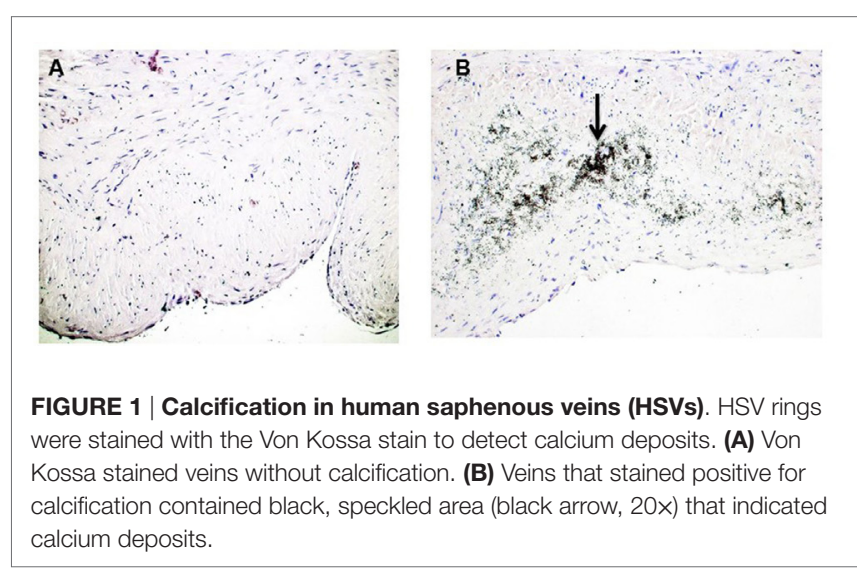



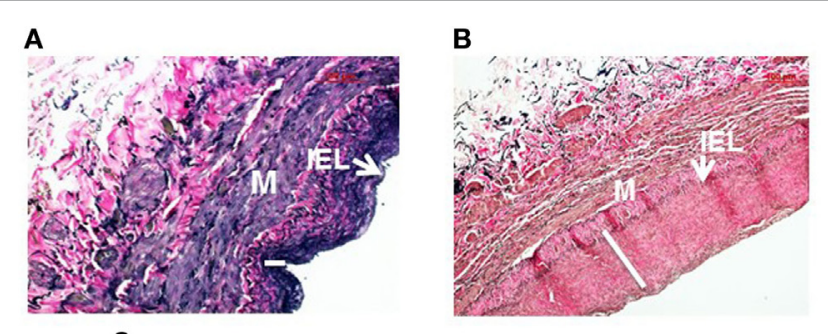

C

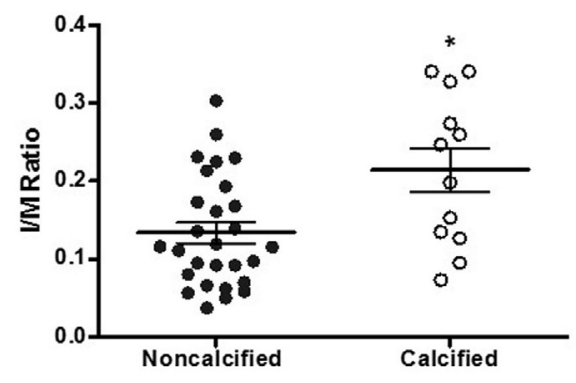

FIGURE 2 | Intimal thickening in human saphenous veins (HSVs) [Verhoeff-Van Gieson (VVG) stain]. HSVs were fixed, sectioned, and stained with WG and imaged with light microscopy. Representative images of non-calcified vein with thin intima [(A), 20x] and calcified vein with thick intima [(B), 20x]. White line indicates the thickness of intima. IEL, internal elastic lamina, M, media. (C) The intima to media (I/M) ratio of calcified veins compared to non-calcified veins was significantly higher $\left({ }^{*} p=0.0058\right.$, $n=12-28)$

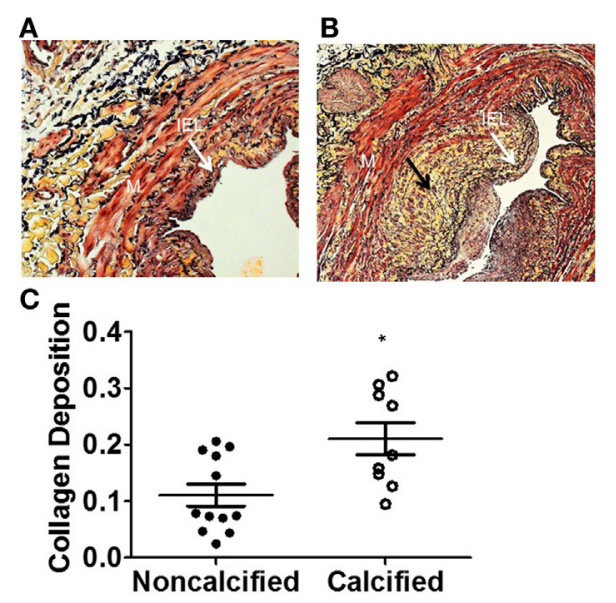

FIGURE 3 | Increased extracellular matrix (ECM) deposition in human saphenous veins with calcification. The amount of ECM deposition was evaluated using the Movat pentachrome stain. The yellow areas (black arrow) represent collagen in non-calcified vein $\mathbf{( A )}$ and calcified vein (B).

(C) Quantitation of collagen. The calcified veins had a significantly higher amount of ECM deposition compared to non-calcified veins $(p=0.0182$, $n=10-12)$.

\section{Patient Age and Kidney Function Predict Venous Calcifications}

Patient data were collected and paired with each vein sample. Patient groups were defined as those without evidence of

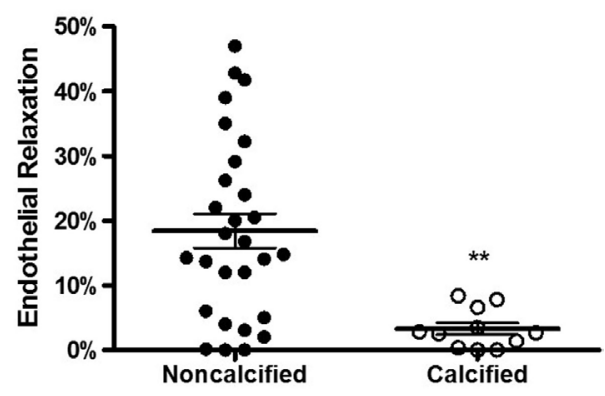

FIGURE 4 | Calcification and endothelial relaxation of human saphenous vein. The vein segments were equilibrated in a muscle bath and contracted with phenylephrine $\left(10^{-6} \mathrm{M}\right)$ and relaxed with carbachol $\left(10^{-7} \mathrm{M}\right)$ to measure the endothelial relaxation. The endothelial relaxation of veins with calcification was significantly lower compared to non-calcified veins $(p=0.0011, n=12-28)$.

venous calcium (non-calcified) and those with any amount of calcium (calcified). Using the Pearson chi-squared test to determine which factors were associated with calcification the following had a strong association with calcification: age, ever smoker, chronic kidney disease (CKD), preoperative serum creatinine (SCr), and glomerular filtration rate. After running a stepwise regression fit using minimum Bayesian information criterion as a stopping rule, age and preoperative $\mathrm{SCr}$ were entered into the model. A nominal logistic regression of these two variables yielded significance for both variables in a multivariate analysis. Preoperative $\mathrm{SCr}$ had an area under the curve of $0.81, p=0.001$, and a unit odds ratio of 405.7, age had an area under the curve of $0.78, p=0.003$, and a unit odds ratio of 1.12 (Figure 5; Table 1).

\section{DISCUSSION}

This is the first study, to our knowledge, to describe venous calcification in terms of concurrent vein pathology, endothelial function, and patient risk factors. In this study, $30 \%$ of the veins analyzed had calcium deposits. Veins with calcium had higher intimal thickness (Figure 2), increased extracellular collagen deposition (Figure 3), and decreased endothelial relaxation (Figure 4). Significant patient risk factors included age and preoperative SCr (Table 1).

Once believed to be a passive process of metabolic derangement, vascular calcification is now thought to result from dedifferentiation of VSMC to an osteoblast phenotype in response to injury. Osteoblasts responsible for bone calcification produce a primarily collagen-based ECM that is subsequently mineralized with hydroxyapatite, a calcium phosphate (16). Arterial calcification studies have demonstrated a similar pattern of collagen and calcium deposition (17). In the present study, we observed calcium deposits within areas of increased ECM collagen with more collagen per area in calcified veins. This suggests the presence of cell-mediated osteogenesis by osteoblast-type VSMC within veins as in arterial and bone calcium deposition. 


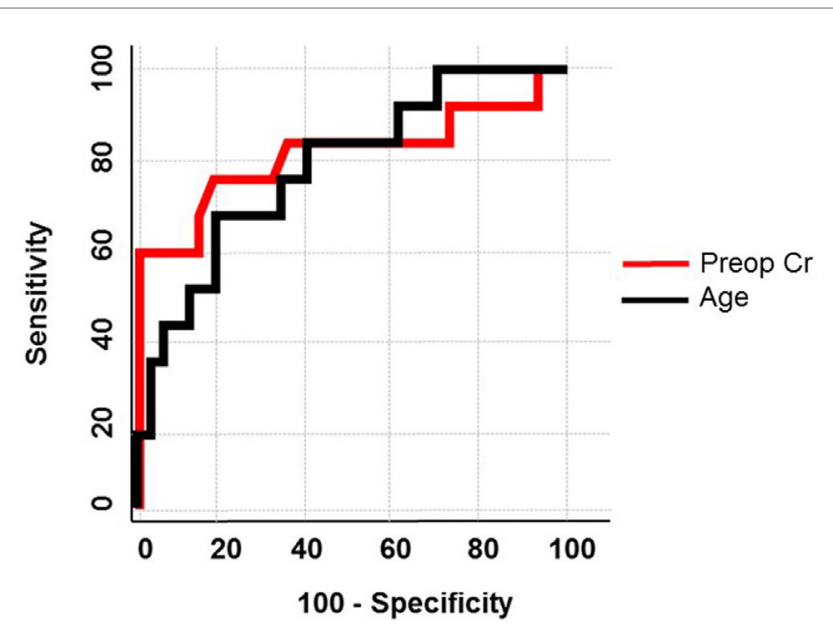

FIGURE 5 | Receiver operator curve using age and preoperative creatinine. In a multivariate logistic regression, age and preoperative creatinine were used to predict calcification of human saphenous veins resulting in an area under the curve of 0.78 and 0.81 , respectively.

The pathological deposition of vascular ECM facilitates not only mineralization but also the migration of VSMCs from media to intima, a key underlying process of IH (18). Chitalia et al. reported on the coexistence of $\mathrm{IH}$ and vascular calcification within brachial and radial arteries of CKD patients (19) while Janda et al. concluded that intimal thickness within the carotid artery predicted the presence and severity of calcification (20). Our study found a strong association $\left(r^{2}=0.184\right)$ between calcium and intimal thickness within the saphenous vein as well. This is of particular importance in assessing vein quality prior to arterial bypass as preexisting intimal thickness in veins predicts bypass graft failure (21).

The ideal venous bypass conduit has a functioning, intact endothelium, the primary regulator of vascular tone, and vessel wall hemostasis (22). The loss of endothelial function predisposes the vessel to vasospasm, platelet aggregation, and IH. In this study, we found that the presence of venous calcification predicted reduced endothelial-dependent relaxation.

The temporal organization of events cannot be concluded from these data. It is likely that an injurious venous environment related to cardiovascular risk factors causes loss of endothelial regulation and VSMC dedifferentiation. This may in turn lead to $\mathrm{IH}$ and calcium deposition. It is also possible that vein calcification preceded the development of IH as can be seen in postphlebitic vein segments or alternatively that endothelial dysfunction related to venous reflux was the primary event with subsequent development of the observed morphological changes. The precise sequence of events in a particular patient or group of patients may be heterogeneous. Sessa et al. demonstrated that saphenous vein endothelial function was reduced in diabetics, smokers, and patients with hypertension (23), while age, diabetes, and end-stage renal disease (ESRD) have long been associated with the development of arterial calcification (24). Not surprisingly, risk factors for saphenous vein calcification in our study were
TABLE 1 | Demographic data on patients with calcium deposits or no calcium deposits within a saphenous vein sample.

\begin{tabular}{|c|c|c|c|}
\hline Patient characteristics & $\begin{array}{c}\text { Non-calcified } \\
\text { vein }(n=33)\end{array}$ & Calcified vein $(n=12)$ & $p$ \\
\hline Age & $60.5 \pm 1.8$ & $72.3 \pm 2.9$ & 0.001 \\
\hline Male sex & $85(\%)$ & $75(\%)$ & 0.456 \\
\hline Caucasian & $88(\%)$ & $100(\%)$ & 0.200 \\
\hline BMl & $30.2 \pm 1.5$ & $26.4 \pm 2.3$ & 0.173 \\
\hline \multicolumn{4}{|l|}{ Smoking status } \\
\hline Current smoker & $42.4(\%)$ & $16.7(\%)$ & 0.101 \\
\hline Former smoker & $39.4(\%)$ & 33.3(\%) & 0.456 \\
\hline Non-smoker & $9.1(\%)$ & $41.6(\%)$ & 0.018 \\
\hline \multicolumn{4}{|l|}{ Comorbidities } \\
\hline Diabetes mellitus & $36.4(\%)$ & $58.3(\%)$ & 0.764 \\
\hline Hypertension & $90.9(\%)$ & $91.7(\%)$ & 0.938 \\
\hline COPD & $9.1(\%)$ & $16.7(\%)$ & 0.485 \\
\hline Hyperlipidemia & $84.8(\%)$ & $66.7(\%)$ & 0.186 \\
\hline Chronic kidney disease & $21.2(\%)$ & $58.3(\%)$ & 0.017 \\
\hline \multicolumn{4}{|l|}{ Medications } \\
\hline ARB & $6.1(\%)$ & $25(\%)$ & 0.077 \\
\hline $\mathrm{ACEi}$ & $60.6(\%)$ & $50(\%)$ & 0.268 \\
\hline Statins & $87.9(\%)$ & $91.7(\%)$ & 0.700 \\
\hline Nitrates & $18.2(\%)$ & 33.3(\%) & 0.290 \\
\hline Beta-blockade & 87.9(\%) & 83.3(\%) & 0.302 \\
\hline CCA & $18.2(\%)$ & $8.3(\%)$ & 0.432 \\
\hline ASA & $90.9(\%)$ & $91.7(\%)$ & 0.486 \\
\hline Other antiplatelet & $(\%)$ & $(\%)$ & \\
\hline \multicolumn{4}{|l|}{ Insulin } \\
\hline \multicolumn{4}{|l|}{ Laboratory values } \\
\hline Total cholesterol & $145.9 \pm$ & $164.3 \pm$ & 0.335 \\
\hline HDL & $36.6 \pm$ & $35.11 \pm$ & 0.702 \\
\hline LDL & $98.3 \pm$ & $84.4 \pm$ & 0.307 \\
\hline Triglycerides & $165.2 \pm$ & $180.6 \pm$ & 1.000 \\
\hline $\mathrm{Hg} \mathrm{A1C}$ & $6.3 \pm$ & $6.3 \pm$ & 0.970 \\
\hline Serum creatinine & $0.9 \pm$ & $1.3 \pm$ & 0.001 \\
\hline
\end{tabular}

Bold font indicates the significant data.

older age and elevated preoperative SCr. While smoking status, ESRD, and hypertension did not reveal a significant association with vein calcification, we suspect a larger sample size would ultimately reveal a trend mirroring arterial calcification risk factors.

There are several limitations with this study. The sample size was relatively small. Only acute variables were examined, and further studies with a larger sample size are needed to draw conclusions regarding venous calcification and long-term bypass patency rates. The innate variability between samples when using human tissue can be vast. The sections of vein analyzed were not implanted and, thus, may not represent the histology and function of grafted tissue. Further efforts using imaging modalities such as vein graft duplex US may allow for longitudinal assessment of calcification and assessment of its long-term effects on patency.

\section{CONCLUSION}

The results of this study demonstrated that preexisting calcification in saphenous vein is a marker of poor conduit quality. HSVs 
with calcification can be characterized as having increased intimal thickness, increased ECM deposition, and reduced endothelialdependent relaxation. Calcified saphenous vein is more common in older patients and those with reduced kidney function. Further studies are needed to determine if long-term graft patency rates are affected by preexisting vein calcification.

\section{AUTHOR CONTRIBUTIONS}

Conception and design: CG, KH, FL, JC-F, CB, RG, and PK. Acquisition of data: SP, CG, KH, AB, FL, and PK. Analysis and interpretation of data and review and final approval: SP, CG, KH,

\section{REFERENCES}

1. Conte MS, Bandyk DF, Clowes AW, Moneta GL, Seely L, Lorenz TJ, et al. Results of PREVENT III: a multicenter, randomized trial of edifoligide for the prevention of vein graft failure in lower extremity bypass surgery. J Vasc Surg (2006) 43:742-751; discussion 751. doi:10.1016/j.jvs.2005. 12.058

2. Alexander JH, Hafley G, Harrington RA, Peterson ED, Ferguson TB Jr, Lorenz TJ, et al. Efficacy and safety of edifoligide, an E2F transcription factor decoy, for prevention of vein graft failure following coronary artery bypass graft surgery: PREVENT IV: a randomized controlled trial. JAMA (2005) 294:2446-54. doi:10.1001/jama.294.19.2446

3. Motwani JG, Topol EJ.Aortocoronary saphenous vein graft disease: pathogenesis, predisposition, and prevention. Circulation (1998) 97:916-31. doi:10.1161/01.CIR.97.9.916

4. Kanellaki-Kyparissi M, Kouzi-Koliakou K, Marinov G, Knyazev V. Histological study of arterial and venous grafts before their use in aortocoronary bypass surgery. Hellenic J Cardiol (2005) 46:21-30.

5. Li FD, Sexton KW, Hocking KM, Osgood MJ, Eagle S, Cheung-Flynn J, et al. Intimal thickness associated with endothelial dysfunction in human vein grafts. J Surg Res (2013) 180:e55-62. doi:10.1016/j.jss.2012.06.017

6. Marin ML, Veith FJ, Panetta TF, Gordon RE, Wengerter KR, Suggs WD, et al. Saphenous vein biopsy: a predictor of vein graft failure. J Vasc Surg (1993) 18:407-414; discussion 414-405. doi:10.1016/0741-5214(93) 90258-N

7. Chowdhury UK, Airan B, Mishra PK, Kothari SS, Subramaniam GK, Ray R, et al. Histopathology and morphometry of radial artery conduits: basic study and clinical application. Ann Thorac Surg (2004) 78:1614-21. doi:10.1016/ j.athoracsur.2004.03.105

8. Kaufer E, Factor SM, Frame R, Brodman RF. Pathology of the radial and internal thoracic arteries used as coronary artery bypass grafts. Ann Thorac Surg (1997) 63:1118-22. doi:10.1016/S0003-4975(96)01393-8

9. Verma S, Szmitko PE, Weisel RD, Bonneau D, Latter D, Errett L, et al. Should radial arteries be used routinely for coronary artery bypass grafting? Circulation (2004) 110:e40-6. doi:10.1161/01.CIR.0000136998. 39371.FF

10. Demer LL, Tintut Y. Vascular calcification: pathobiology of a multifaceted disease. Circulation (2008) 117:2938-48. doi:10.1161/CIRCULATIONAHA. 107.743161

11. Sinha S, Eddington H, Kalra PA. Vascular calcification: lessons from scientific models. J Ren Care (2009) 35(Suppl 1):51-6. doi:10.1111/ j.1755-6686.2009.00065.x

12. Chen NX, Moe SM. Vascular calcification: pathophysiology and risk factors. Curr Hypertens Rep (2012) 14:228-37. doi:10.1007/s11906-0120265-8

13. Zoccali C, Bolignano D, D’Arrigo G, Dekker FW, Fliser D, Heine GH, et al. Validity of vascular calcification as a screening tool and as a surrogate end point in clinical research. Hypertension (2015) 66:3-9. doi:10.1161/ HYPERTENSIONAHA.115.04801
AB, FL, JC-F, RG, CB, and PK. Drafting of manuscript: SP, CG, and $\mathrm{PK}$.

\section{FUNDING}

This material is based on the work supported in part by the Department of Veterans Affairs, Veterans Health Administration, Biomedical Laboratory Research and Development Grant $101 B X 002036$ and a National Institute of Health grant RO1HL70715 to CB. This material is the result of work supported in part with resources and the use of facilities at the VA Tennessee Valley Healthcare System.

14. Hocking KM, Brophy C, Rizvi SZ, Komalavilas P, Eagle S, Leacche M, et al Detrimental effects of mechanical stretch on smooth muscle function in saphenous veins. J Vasc Surg (2011) 53:454-60. doi:10.1016/j.jvs.2010.09.010

15. Newby AC, Zaltsman AB. Molecular mechanisms in intimal hyperplasia. J Pathol (2000) 190:300-9. doi:10.1002/(SICI)1096-9896(200002) 190:3<300::AID-PATH596>3.3.CO;2-9

16. Fedarko NS, Moerike M, Brenner R, Robey PG, Vetter U. Extracellular matrix formation by osteoblasts from patients with osteogenesis imperfecta. J Bone Miner Res (1992) 7:921-30. doi:10.1002/jbmr.5650070809

17. Moe SM, O'Neill KD, Duan D, Ahmed S, Chen NX, Leapman SB, et al. Medial artery calcification in ESRD patients is associated with deposition of bone matrix proteins. Kidney Int (2002) 61:638-47. doi:10.1046/j.1523-1755. 2002.00170.x

18. Galis ZS, Khatri JJ. Matrix metalloproteinases in vascular remodeling and atherogenesis: the good, the bad, and the ugly. Circ Res (2002) 90:251-62.

19. Chitalia N, Ross L, Krishnamoorthy M, Kapustin A, Shanahan CM, Kaski JC, et al. Neointimal hyperplasia and calcification in medium sized arteries in adult patients with chronic kidney disease. Semin Dial (2015) 28:E35-40. doi:10.1111/sdi.12335

20. Janda K, Krzanowski M, Gajda M, Dumnicka P, Fedak D, Lis GJ, et al. Cardiovascular risk in chronic kidney disease patients: intima-media thickness predicts the incidence and severity of histologically assessed medial calcification in radial arteries. BMC Nephrol (2015) 16:78. doi:10.1186/ s12882-015-0067-8

21. Kouzi-Koliakos K, Kanellaki-Kyparissi M, Marinov G, Knyazhev V, Tsalie E, Batzios C, et al. Prebypass histological and ultrastructural evaluation of the long saphenous vein as a predictor of early graft failure. Cardiovasc Pathol (2006) 15:336-46. doi:10.1016/j.carpath.2006.07.005

22. Gür D, Gür Ö, Gürkan S, Cömez S, Gönültaş A, Yılmaz M. Comparison of endothelial function of coronary artery bypass grafts in diabetic and nondiabetic patients: which graft offers the best? Anatol J Cardiol (2015) 15:657-62. doi:10.5152/akd.2014.5613

23. Sessa C, Morasch MD, Friedland M, Kline RA. Risk factors of atherosclerosis and saphenous vein endothelial function. Int Angiol (2001) 20:152-63.

24. Lanzer P, Boehm M, Sorribas V, Thiriet M, Janzen J, Zeller T, et al. Medial vascular calcification revisited: review and perspectives. Eur Heart J (2014) 35:1515-25. doi:10.1093/eurheartj/ehu163

Conflict of Interest Statement: The authors declare that the research was conducted in the absence of any commercial or financial relationships that could be construed as a potential conflict of interest.

Copyright (c) 2017 Pedigo, Guth, Hocking, Banathy, Li, Cheung-Flynn, Brophy, Guzman and Komalavilas. This is an open-access article distributed under the terms of the Creative Commons Attribution License (CC BY). The use, distribution or reproduction in other forums is permitted, provided the original author $(s)$ or licensor are credited and that the original publication in this journal is cited, in accordance with accepted academic practice. No use, distribution or reproduction is permitted which does not comply with these terms. 